\title{
Emphysema and bronchiectasis in COPD patients with previous pulmonary tuberculosis: computed tomography features and clinical implications
}

This article was published in the following Dove Press journal: International Journal of COPD

\author{
Jianmin Jin' \\ Shuling $\mathrm{Li}^{2}$ \\ Wenling $\mathrm{Yu}^{2}$ \\ Xiaofang Liu' \\ Yongchang Sun ${ }^{1,3}$ \\ 'Department of Respiratory and \\ Critical Care Medicine, Beijing \\ Tongren Hospital, Capital Medical \\ University, Beijing, ${ }^{2}$ Department of \\ Radiology, Beijing Tongren Hospital, \\ Capital Medical University, Beijing, \\ ${ }^{3}$ Department of Respiratory \\ and Critical Care Medicine, \\ Peking University Third Hospital, \\ Beijing, China
}

\begin{abstract}
Background: Pulmonary tuberculosis (PTB) is a risk factor for COPD, but the clinical characteristics and the chest imaging features (emphysema and bronchiectasis) of COPD with previous PTB have not been studied well.

Methods: The presence, distribution, and severity of emphysema and bronchiectasis in COPD patients with and without previous PTB were evaluated by high-resolution computed tomography (HRCT) and compared. Demographic data, respiratory symptoms, lung function, and sputum culture of Pseudomonas aeruginosa were also compared between patients with and without previous PTB. Results: A total of 231 COPD patients $(82.2 \%$ ex- or current smokers, $67.5 \%$ male) were consecutively enrolled. Patients with previous PTB (45.0\%) had more severe $(p=0.045)$ and longer history $(p=0.008)$ of dyspnea, more exacerbations in the previous year $(p=0.011)$, and more positive culture of $P$. aeruginosa ( $p=0.001$ ), compared with those without PTB. Patients with previous PTB showed a higher prevalence of bronchiectasis $(p<0.001)$, which was more significant in lungs with tuberculosis (TB) lesions, and a higher percentage of more severe bronchiectasis (Bhalla score $\geq 2, p=0.031$ ), compared with those without previous PTB. The overall prevalence of emphysema was not different between patients with and without previous PTB, but in those with previous PTB, a higher number of subjects with middle ( $p=0.001)$ and lower ( $p=0.019)$ lobe emphysema, higher severity score $(p=0.028)$, higher prevalence of panlobular emphysema ( $p=0.013)$, and more extensive centrilobular emphysema $(p=0.039)$ were observed. Notably, in patients with TB lesions localized in a single lung, no difference was found in the occurrence and severity of emphysema between the 2 lungs.

Conclusion: COPD patients with previous PTB had unique features of bronchiectasis and emphysema on HRCT, which were associated with significant dyspnea and higher frequency of severe exacerbations. While PTB may have a local effect on bronchiectasis, its involvement in airspace damage in COPD may be extensive, probably through interactions with cigarette smoke.
\end{abstract}

Keywords: chronic obstructive pulmonary disease, tuberculosis, bronchiectasis, emphysema

\section{Background}

Both COPD and tuberculosis (TB) are leading causes of morbidity and mortality in developing countries. ${ }^{1,2}$ The two diseases not only share common risk factors such as smoking and poor socioeconomic status, but also coexist and interact in disease development and manifestation. ${ }^{1-3}$ Although pulmonary TB (PTB) has been identified as a risk factor for $\mathrm{COPD},{ }^{4}$ and several studies have found an association between previous PTB and higher COPD prevalence, ${ }^{5-7}$ few studies have explored the clinical and radiological features of COPD in patients with previous PTB.
Correspondence: Yongchang Sun Department of Respiratory and Critical Care Medicine, Beijing Tongren Hospital, Capital Medical University, I Dong Jiao Min Xiang, Dongcheng District, Beijing 100730, China

Tel +86 I56 II 963697

Email suny@bjmu.edu.cn (c) (1) (5) 2018 jin et al. This work is published and licensed by Dove Medical Press Limited. The full terms of this license are available at https://www.dovepress.com/terms.php cc) hereby accept the Terms. Non-commercial uses of the work are permitted without any further permission from Dove Medical Press Limited, provided the work is properly attributed. For permission for commercial use of this work, please see paragraphs 4.2 and 5 of our Terms (https://www.dovepress.com/terms.php). 
The chronic inflammatory response and long-term anatomic alterations induced by PTB were believed to be the main pathological basis for the impairment of lung function and poor prognosis. ${ }^{8}$ TB-associated structural alterations, such as scar formation, bronchial stenosis, and bronchiectasis, ${ }^{9}$ affect both the development of COPD and disease manifestation, which may have unique features compared with COPD without previous PTB. Recently, radiological phenotypes of COPD have received much attention, and the presence of pulmonary emphysema, bronchial wall thickening, and bronchiectasis have been proposed as the 3 main morphological findings likely to provide relevant information about different phenotypes of COPD. ${ }^{10}$ Interestingly, both emphysema and bronchiectasis were associated with PTB.

Therefore, we set out to explore whether the prevalence, distribution, and severity of emphysema and bronchiectasis are different, and associated with clinical features in COPD patients with radiological signs of previous PTB. Our results showed that compared with those without PTB, COPD patients with previous PTB demonstrated more prevalent and severe bronchiectasis, and the unique distribution of emphysema, which was associated with significant dyspnea and higher frequency of severe exacerbations.

\section{Materials and methods Study subjects}

Patients with stable COPD visiting Beijing Tongren Hospital, Capital Medical University from July 2008 to July 2016 were evaluated. The diagnosis of COPD was based on the Global Initiative for Chronic Obstructive Lung Disease (GOLD) criteria, ${ }^{4}$ as used in our previous study. These were as follows: ${ }^{11} 1$ ) age $>40$ years, 2) history of smoking (smoking index $>10$ pack-year) and/or exposure to biomass fuel (mostly coal or wood burning stoves)/chemical agents/ noxious particles for $>10$ years, 3 ) chronic cough with or without wheeze for $>3$ months in each year for 2 consecutive years, and 4) irreversible obstructive dysfunction defined as postbronchodilator forced expiratory volume in 1 second $\left(\mathrm{FEV}_{1}\right)$ /forced vital capacity (FVC) $<70 \%$ on spirometry. Patients were excluded if they met any of the following conditions: receiving therapy of systemic corticosteroids or other immunosuppressive agents in the preceding 8 weeks; with allergic bronchopulmonary mycosis, interstitial lung diseases, active PTB, autoimmune diseases, and severe heart failure; doctor-diagnosed asthma or bronchiectasis before diagnosis of COPD; and previous PTB resulting in severe lung damage ("destroyed lung"), since "destroyed lung" per se could lead to severe impairment of lung functions, restrictive and/or obstructive, which could not be differentiated from COPD.

\section{Definition of previous PTB}

Previous PTB was defined as present if discrete linear or reticular fibrotic scars, or dense nodules with distinct margins were identified within the upper lobes and/or superior segment of lower lobes on computed tomography (CT) scan, with or without calcification of the lesions and/or local lymph nodes. ${ }^{11-13}$ A distinct history of TB was not necessary for the determination.

\section{Lung high-resolution computed tomography (HRCT) and evaluation of bronchiectasis and emphysema}

Chest HRCT was performed with a 64-row, multiple detector CT scanner (Philips Company, Amsterdam, the Netherlands). The presence, extent (Smith score), severity (Bhalla score) and types (classified into cylindric, cystic, or mixed) of bronchiectasis, as well as the presence, severity (Goddard score) and types (classified into centrilobular, panlobular, paraseptal, and bullae) of emphysema were evaluated by 2 radiologists experienced in the interpretation of HRCT and blinded to the patients' clinical data. The radiologists finished the evaluations independently and differences in the readings were resolved by their final consensus. ${ }^{11}$

Bronchiectasis was determined to be present if HRCT showed bronchial wall thickening with the ratio of the diameter of bronchus to that of the accompanying pulmonary artery being $>1.1$ (signet ring sign) or the lack of tapering of bronchi (tramline sign). ${ }^{14}$ The extent of bronchiectasis was scored for each pulmonary lobe, with the lingula as a separate lobe. The grading system proposed by Smith et a ${ }^{15}$ was adopted in our study. According to this system, the absence of bronchiectasis was scored as 0 , bronchiectasis in $<25 \%$ of bronchi as 1 , in $25 \%$ to $49 \%$ of bronchi as 2 , in $50 \%$ to $74 \%$ as 3 , and in $\geq 75 \%$ as 4 . The total score ranged from 0 to 24 points. Patients with a score $\leq 1$ were considered as normal, since mild bronchiectasis only visible in a single pulmonary segment may exist in a significant percentage of healthy population. ${ }^{11,16}$ The type of bronchiectasis was identified as cylindric, cystic, or mixed (varicose) according to the morphological characteristics of the dilated bronchi. ${ }^{14}$ The severity of bronchiectasis in each lobe was graded by the Bhalla scoring system on a scale of 0 to $3,{ }^{17,18}$ with 0 for no involvement; 1 for mild, with luminal diameter slightly greater than that of adjacent blood vessel; 2 for moderate, with lumen 2-3 times that of adjacent vessel; and 3 for severe, with lumen 3 times that of adjacent vessel. 
The presence and the type of emphysema were visually assessed for each lobe, with the lingula as a separate lobe. Emphysema was classified into centrilobular, panlobular (panacinar), paraseptal, and bullous as previously reported. ${ }^{14}$ With the presence scored as 1 and the absence scored as 0 , a semi-quantitative method, with scores of both lungs ranging from 0 to 6 , was adopted to show the extent of a certain type of emphysema. The severity of emphysema was visually evaluated with the modified Goddard scoring system. ${ }^{19,20}$ Six images were analyzed in 3 slices (including the aortic arch, carina, and 1-2 $\mathrm{cm}$ above the highest hemidiaphragm) of both lungs, and a total score of all images was considered as a representative value of the severity of emphysema for each patient. Each image was classified as normal (score 0 ), $5 \%$ affected (score 0.5 ), 25\% affected (score 1), $50 \%$ affected (score 2), $75 \%$ affected (score 3 ), and $>75 \%$ affected (score 4); therefore, the total score of each patient ranged from 0 to 24 points.

\section{Pulmonary function tests}

Spirometry (JAEGER, MasterScreen-body + diffusion + APS, Hoechberg, Germany) was performed to determine the lung function measurements and bronchodilator reversibility as we previously reported. ${ }^{11}$ Post-bronchodilator $\mathrm{FEV}_{1} / \mathrm{FVC} \%$ and $\mathrm{FEV}_{1}$ were measured 15 minutes after the inhalation of $400 \mu \mathrm{g}$ salbutamol.

\section{Definition of respiratory symptoms and exacerbations}

Chronic cough and expectoration were considered to be present if the symptoms lasted for more than 3 consecutive months. Exertional dyspnea was identified as present if the mMRC (modified Medical Research Council Questionnaire) score was $\geq 1$. The duration of a symptom such as dyspnea was defined as the length of time from its onset to enrollment of the patient in the study, as we previously described. ${ }^{11}$ Frequency of acute exacerbation (AE) was defined based on the number of times the patient was hospitalized or presented for emergency visits because of AE during the year before enrollment.

\section{Bacterial culture of sputum samples}

Results of sputum cultures were reviewed retrospectively and positive results for bacteria, especially Pseudomonas aeruginosa, were compared between groups as described previously. ${ }^{11}$ The sputum samples were obtained when the patients experienced a severe exacerbation. Sputum samples were acceptable if they contained fewer than 10 squamous epithelial cells and more than 25 leukocytes per low-powered field. ${ }^{11,17}$

\section{Analysis}

The prevalence, distribution, and severity of emphysema and bronchiectasis on HRCT, and the clinical characteristics were compared between COPD patients with and without previous PTB. To explore whether the potential effect of previous PTB on emphysema and bronchiectasis was localized or extensive, we also compared the presence and the severity of emphysema and bronchiectasis between the lung with TB sequelae and the contralateral lung in patients with TB sequelae in a single lung.

The statistical package SPSS version 17.0 (SPSS, Chicago, IL, USA) was used for statistical analysis. Data were expressed as mean $\pm \mathrm{SD}$. Independent-samples $t$-Test (for normal distribution parameters) and Mann-Whitney $U$-test (for abnormal distribution parameters) were adopted for comparisons of continuous data between the 2 groups. Comparisons of continuous data among 3 groups were performed by analysis of variance test (for normal distribution) or Kruskal-Wallis test (for abnormal distribution). Categorical variables between different groups were analyzed by $\chi^{2}$ test. $p$-values $<0.05$ were considered as statistically significant. As for multiple comparisons among 3 or 4 groups (including $\chi^{2}$ test or Kruskal-Wallis test), $p$-values $<0.017$ or $p$-values $<0.008$, respectively, were taken as statistically significant.

\section{Results \\ Clinical characteristics and chest CT features of subjects with COPD}

During the study period, 231 patients with COPD were consecutively enrolled. These included 156 males (67.5\%) and 75 females (32.5\%) with a mean age of 76.8 years, and a majority of them were smokers (82.2\%). The GOLD spirometry classification ranged from 2 to 4 , with 83 patients $(35.9 \%)$ in GOLD 2, 109 (47.2\%) in GOLD 3, and $39(16.9 \%)$ in GOLD 4. Data are shown in Table 1.

Signs of previous PTB were found by chest HRCT in 104 patients $(104 / 231,45.0 \%)$, of whom only 42 (42/231, $18.2 \%)$ had a definite history of PTB and had received anti-TB therapy. TB sequelae in the upper lobes were found in most of the patients $(99 / 104,95.2 \%)$. As for the symptoms, the average time course of chronic cough/expectoration and that of exertional dyspnea were 19.1 and 6.28 years, respectively. $P$. aeruginosa was found positive in the sputum of 15 patients with COPD, and 13 of them had coexistent bronchiectasis on CT (Table 1). 
Table I Baseline and clinical characteristics of subjects with COPD, with and without PTB

\begin{tabular}{|c|c|c|c|c|}
\hline Parameter & COPD & $\begin{array}{l}\text { COPD with } \\
\text { previous TB }\end{array}$ & $\begin{array}{l}\text { COPD without } \\
\text { previous TB }\end{array}$ & $p$-value \\
\hline Subjects, n (\%) & 231 & $104(45.0)$ & $127(55.0)$ & \\
\hline Male, n (\%) & $156(67.5)$ & $73(70.2)$ & $83(65.4)$ & 0.435 \\
\hline Age, (years) & $76.8(9.4)$ & $77.5(9.4)$ & $76.1(9.3)$ & 0.247 \\
\hline \multicolumn{5}{|l|}{ Smoking status } \\
\hline Present smokers, n (\%) & $68(29.4)$ & $29(27.9)$ & $39(30.7)$ & 0.823 \\
\hline Former smokers, n (\%) & $122(52.8)$ & $55(52.9)$ & $67(52.8)$ & \\
\hline Non-smokers, n (\%) & $4 I(17.7)$ & $20(19.2)$ & $21(16.5)$ & \\
\hline Pack-years smoked & $42.6(25.3)$ & $45.3(26.4)$ & $40.5(24.4)$ & 0.187 \\
\hline Body mass index, $\left(\mathrm{kg} / \mathrm{m}^{2}\right)$ & $23.2(4.77)$ & $22.8(4.33)$ & $23.6(5.08)$ & 0.186 \\
\hline History of chronic cough/sputum, (years) & $19.1(12.3)$ & $20.1(12.2)$ & $18.3(12.5)$ & 0.182 \\
\hline History of dyspnea, (years) & $6.28(5.70)$ & $7.31(6.19)$ & $5.44(5.13)$ & 0.008 \\
\hline \multicolumn{5}{|l|}{ Daily treatments, n (\%) } \\
\hline Anticholinergic & $160(69.2)$ & $69(66.3)$ & $91(71.6)$ & 0.384 \\
\hline Long-acting $\beta$ adrenergic & $143(61.9)$ & $62(59.6)$ & 81 (63.7) & 0.517 \\
\hline ICS & $143(61.9)$ & $62(59.6)$ & 81 (63.7) & 0.517 \\
\hline $\mathrm{mMRC}$ & $2.46(1.15)$ & $2.63(1.16)$ & $2.33(1.13)$ & 0.045 \\
\hline $\mathrm{mMRC} \geq 2, \mathrm{n}(\%)$ & $183(79.2)$ & $89(85.6)$ & $94(74.0)$ & 0.031 \\
\hline Exacerbations in the previous year & $1.30(1.27)$ & $1.56(1.39)$ & $1.09(1.12)$ & 0.011 \\
\hline Hemoglobin, (g/L) & I25.I (I7.I) & I24.8 (I6.3) & I25.4 (I7.8) & 0.783 \\
\hline $\mathrm{FEV}_{1} / \mathrm{FVC}, \%$ & $0.46(0.11)$ & $0.45(0.10)$ & $0.47(0.11)$ & 0.252 \\
\hline $\mathrm{FEV}, \%$ predicted & $0.43(0.13)$ & $0.4 \mathrm{I}(0.12)$ & $0.44(0.14)$ & 0.215 \\
\hline \multicolumn{5}{|l|}{ GOLD grading, n (\%) } \\
\hline Grade II & $83(35.9)$ & $37(35.6)$ & $46(36.3)$ & 0.987 \\
\hline Grade III & $109(47.2)$ & $49(47.1)$ & $60(47.2)$ & \\
\hline Grade IV & $39(16.9)$ & $18(17.3)$ & $21(16.5)$ & \\
\hline \multicolumn{5}{|l|}{ Location of PTB sequelae } \\
\hline Upper lobes, n (\%) & & $99(95.2)$ & $0(0)$ & \\
\hline Middle lobes, n (\%) & & $2(1.92)$ & $0(0)$ & \\
\hline Lower lobes, n (\%) & & $8(7.69)$ & $0(0)$ & \\
\hline Positive culture of bacteria, $\mathrm{n}(\%)$ & $53(22.9)$ & $29(27.9)$ & $24(18.9)$ & 0.106 \\
\hline Positive culture of Pseudomonas aeruginosa, $\mathrm{n}(\%)$ & $15(6.5)$ & $13(12.5)$ & $2(1.6)$ & 0.001 \\
\hline
\end{tabular}

Notes: $p$-values represent the comparisons between COPD patients with TB and those without. Lingular segment of left lung was taken as "middle lobe" of left lung. Continuous data are expressed as mean (standard deviation), Categorical data are expressed as number (percentage). The statistically significance values $p<0.05$ shown in bold.

Abbreviations: PTB, pulmonary tuberculosis; TB, tuberculosis; ICS, inhaled corticosteroids; mMRC, modified Medical Research Council Questionnaire; FEV , forced expiratory volume in I second; FVC, forced vital capacity; GOLD, Global Initiative for Chronic Obstructive Lung Disease.

Bronchiectasis on HRCT was found in 50.6\% (117/231) of the COPD patients. With regard to the sites of bronchiectasis, the middle lobes/lingular segments $(64.1 \%, p=0.018)$, and the lower lobes $(68.3 \%, p=0.002)$ were involved more often compared with the upper lobes (48.7\%). No statistical difference was found in the Smith score among upper lobes, middle lobes/lingular segments, and lower lobes (3.45, 3.96, and 4.02, respectively, $p=0.321$ ). Among COPD patients with comorbid bronchiectasis (COPD-Bx, $\mathrm{n}=117$ ), most showed mild bronchiectasis (Bhalla score $=1$ ), and only $6.8 \%(8 / 117)$ of them had a Bhalla score $\geq 2$. The majority $(115 / 117$, 98.3\%) of the COPD-Bx subjects demonstrated cylindric bronchiectasis, and mixed (varicose) type was found in only 4 patients $(4 / 117,3.4 \%)$, with no patients showing typical cystic bronchiectasis (Table 2).
Emphysema on HRCT was found in $62.3 \%$ (144/231) of COPD patients. With regard to the sites of emphysema, the upper lobes (92.4\%) were involved more often compared with the middle lobes/lingular segments (68.7\%), and the lower lobes (63.1\%); both $p<0.001$. In addition, a significant difference was found in the severity of emphysema (Goddard score) among the upper, middle, and lower lobes (overall $p=0.015$ ). The Goddard score of the upper lobes was found to be significantly higher than that of the lower lobes $(p=0.006)$. With regard to the types of emphysema, centrilobular emphysema was the most common (42\%, 97/231), followed by panlobular $(34.6 \%, 80 / 231)$, bullous $(26.8 \%, 62 / 231)$, and paraseptal emphysema $(19.9 \%, 46 / 231)$. According to the semi-quantitative evaluation, the distribution of centrilobular or panlobular emphysema was found to be more extensive 
Table 2 CT characteristics of bronchiectasis and emphysema of subjects with COPD, with and without PTB

\begin{tabular}{|c|c|c|c|c|}
\hline Parameters & $\begin{array}{l}\text { COPD } \\
(n=231)\end{array}$ & $\begin{array}{l}\text { COPD with } \\
\text { previous TB } \\
(n=104)\end{array}$ & $\begin{array}{l}\text { COPD without } \\
\text { previous TB } \\
(n=\mid 27)\end{array}$ & $p$-value \\
\hline Bronchiectasis prevalence, $\mathrm{n}(\%)$ & $117(50.6)$ & $67(64.4)$ & $50(39.4)$ & 0.000 \\
\hline Upper lobes, n (\%) & 57 (48.7) & $42(62.7)$ & $15(30.0)$ & 0.000 \\
\hline Middle lobes, n (\%) & $75(64.1)$ & $45(67.2)$ & $30(60.0)$ & 0.424 \\
\hline Lower lobes, n (\%) & $80(68.3)$ & $42(62.6)$ & $38(76.0)$ & 0.126 \\
\hline Smith score $\mathrm{e}^{\mathrm{a}}$ & $6.97(5.8 \mathrm{I})$ & $7.78(6.29)$ & $5.90(4.96)$ & 0.162 \\
\hline Upper lobes & $3.45(2.05)$ & $3.73(2.66)$ & $2.66(1.23)$ & 0.194 \\
\hline Middle lobes & $3.96(2.31)$ & $4.06(2.3 I)$ & $3.80(2.32)$ & 0.541 \\
\hline Lower lobes & $4.02(2.3 \mathrm{I})$ & $4.31(2.34)$ & $3.71(2.26)$ & 0.246 \\
\hline \multicolumn{5}{|l|}{ Bhalla score ${ }^{b}$} \\
\hline I, n (\%) & $109(93.2)$ & $59(88.0)$ & $50(100.0)$ & 0.031 \\
\hline$\geq 2, \mathrm{n}(\%)$ & $8(6.8)$ & $8(11.9)$ & $0(0)$ & \\
\hline \multicolumn{5}{|l|}{ Bronchiectasis type } \\
\hline Cylindric & $115(98.3)$ & $65(97.0)$ & $50(100)$ & \\
\hline Cystic & $0(0)$ & $0(0)$ & $0(0)$ & \\
\hline Varicose & $4(3.4)$ & $4(5.9)$ & $0(0)$ & \\
\hline Emphysema prevalence, n (\%) & $144(62.3)$ & $70(67.3)$ & $74(58.2)$ & 0.158 \\
\hline Upper lobes, n (\%) & $133(92.4)$ & $67(95.7)$ & $66(89.1)$ & 0.141 \\
\hline Middle lobes, n (\%) & 99 (68.7) & $57(81.4)$ & $42(56.7)$ & 0.001 \\
\hline Lower lobes, n (\%) & $91(63.1)$ & $51(72.8)$ & $40(54.1)$ & 0.019 \\
\hline Goddard score $\mathrm{a}^{\mathrm{a}}$ & $7.00(5.66)$ & $7.88(5.5 \mathrm{I})$ & $6.17(5.7 I)$ & 0.028 \\
\hline Upper levels & $3.57(2.28)$ & $3.59(2.26)$ & $3.56(2.33)$ & 0.940 \\
\hline Middle levels & $2.92(1.89)$ & $2.82(I .8 I)$ & $3.05(2.00)$ & 0.684 \\
\hline Lower levels & $2.68(1.88)$ & $2.95(1.99)$ & $2.33(1.69)$ & 0.120 \\
\hline \multicolumn{5}{|l|}{ Types of emphysema, prevalence } \\
\hline Centrilobular, n (\%) & $97(42.0)$ & $49(47.1)$ & $48(37.8)$ & 0.153 \\
\hline Panlobular, n (\%) & $80(34.6)$ & $45(43.2)$ & $35(27.5)$ & 0.013 \\
\hline Paraseptal, n (\%) & $46(19.9)$ & $24(23.0)$ & $22(17.3)$ & 0.276 \\
\hline Bullae, n (\%) & $62(26.8)$ & $34(32.7)$ & $28(22.0)$ & 0.069 \\
\hline \multicolumn{5}{|l|}{ Types of emphysema, score ${ }^{a}$} \\
\hline Centrilobular & $2.76(1.59)$ & $3.12(1.7 I)$ & 2.40 (1.39) & 0.039 \\
\hline Panlobular & $2.93(1.7 I)$ & $2.78(1.67)$ & $3.14(1.76)$ & 0.322 \\
\hline Paraseptal & I.7I (0.88) & I.7I (0.80) & $1.73(0.98)$ & 0.943 \\
\hline Bullae & $1.90(1.11)$ & 1.94 (I.07) & $1.86(1.17)$ & 0.518 \\
\hline
\end{tabular}

Notes: aSmith score, Goddard score, and semi-quantitative score of different types of emphysema represent the means of patients with bronchiectasis, or with emphysema, or with a certain type of emphysema. 'Defined based on the highest Bhalla score of the patient. Lingular segment of the left lung was taken as "middle lobe" of the left lung. $p$-values represent the comparisons between COPD patients with TB and those without. Continuous data are expressed as mean (standard deviation), Categorical data are expressed as number (percentage). The statistically significance values $p<0.05$ shown in bold.

Abbreviations: PTB, pulmonary tuberculosis; TB, tuberculosis.

than that of paraseptal or bullae emphysema (2.76 vs 1.71 and 2.76 vs $1.90,2.93$ vs 1.71 and 2.93 vs 1.90 , respectively, all the $p$-values $<0.001$ ) (Table 2).

\section{Clinical characteristics of COPD patients with previous PTB}

No statistical difference was found in age, gender, smoking status, body mass index, maintenance medication, $\mathrm{FEV}_{1} \%$ predicted, and GOLD grading between COPD patients with and without previous infection of PTB. However, patients with previous PTB showed a longer history of dyspnea (7.31 vs 5.44 years), more severe dyspnea (mMRC: 2.63 vs 2.33 ; $m M R C \geq 2: 85.6 \%$ vs $74.0 \%$ ), a higher frequency of exacerbation in the previous year (1.56 vs 1.09), and a higher positive rate of $P$. aeruginosa by sputum culture $(12.5 \%$ vs $1.6 \%)$ (Table 1).

\section{Characteristics of bronchiectasis in COPD patients with previous PTB}

The percentage of patients with $\mathrm{CT}$ bronchiectasis was higher in those with previous PTB (64.4\%) compared with those without PTB (39.4\%, $p<0.001)$. The difference was more significant when the upper lobes were compared (62.7\% vs $30.0 \%, p<0.001$ ) (Table 2, Figures 1 and 3). Coexisting upper lobe bronchiectasis was found in $42.4 \%$ (42/99) of patients with PTB sequelae in upper lobes, but in only 


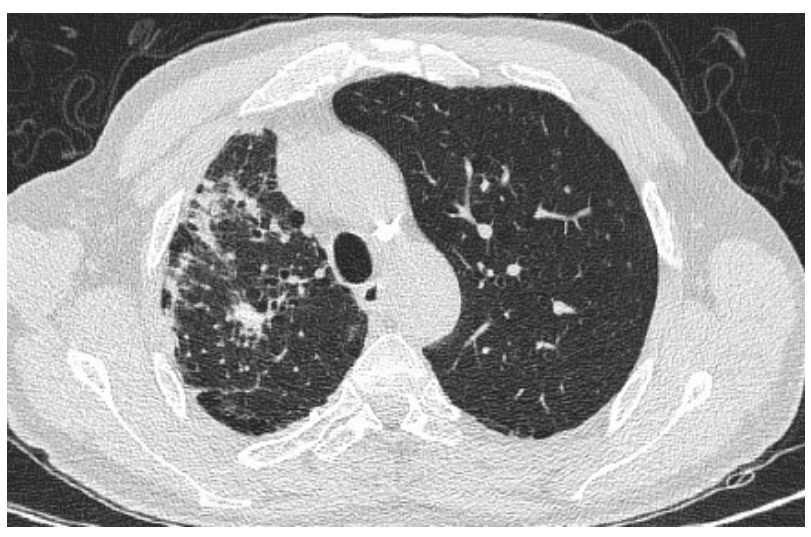

Figure I Bronchiectasis in case I with TB sequelae in RUL (HRCT).

Abbreviations: TB, tuberculosis; RUL, right upper lobe; HRCT, high-resolution CT, computed tomography.

$11.8 \%(15 / 127)$ of patients without PTB sequelae $(p<0.001)$. For patients with bronchiectasis, the Smith score tended to be higher in those with previous PTB $(\mathrm{n}=67)$ compared with those without ( $\mathrm{n}=50$ ), though the difference was not significant. The severity of bronchiectasis was identified as mild (Bhalla score $=1$ ) and cylindric for most of the patients in the 2 groups, but more severe (Bhalla score $\geq 2$ ) or varicose bronchiectasis was found only in COPD patients with previous PTB (Table 2).

Presence and characteristics of bronchiectasis were also compared between the lung with TB sequelae and the contralateral lung in patients with TB sequelae limited to a single lung $(\mathrm{n}=65)$. The prevalence of bronchiectasis in the TB-involved lung, especially the upper lobe, was significantly higher $(p<0.01)$ compared with the contralateral lung (Table 3, Figures 1 and 3), but no difference was found in the severity of bronchiectasis (Table 3).

\section{Characteristics of emphysema in COPD patients with previous PTB}

The prevalence of emphysema between patients with and without previous PTB showed no significant difference (67.3\% vs $58.2 \%)$. Interestingly, in patients with previous PTB, more had emphysema of the middle lobes/lingular segments $(81.4 \%$ vs $56.7 \%, p=0.001)$ and of the lower lobes $(72.8 \%$ vs $54.1 \%, p=0.019)$, compared with those without PTB, but no difference was found in the upper lobe emphysema. The severity score of emphysema (Goddard score) in patients with previous PTB was higher than that in patients without PTB ( 7.88 vs $6.17, p=0.028)$. Patients with previous PTB also demonstrated a statistically higher prevalence of panlobular emphysema ( $43.2 \%$ vs $27.5 \%, p=0.013$ ) and bullae $(32.7 \%$ vs $22 \%, p=0.069)$. The distribution of centrilobular emphysema in patients with PTB was more extensive than that in patients without PTB (3.12 vs 2.40, $p=0.039$ ) (Table 2).

Presence and characteristics of emphysema were also compared between the lung with TB sequelae and the contralateral lung in patients with TB sequelae limited to a single lung $(\mathrm{n}=65)$. Interestingly, no significant difference was seen in the prevalence, severity, and type of emphysema between the 2 lungs of the same patients. (Table 3, Figures 2 and 3).

\section{Discussion}

In this cross-sectional study of the chest imaging (bronchiectasis and emphysema) and clinical features of COPD with previous PTB, the most important finding was that, compared with those without PTB, COPD patients with

Table 3 Bronchiectasis and emphysema of 2 lungs in 65 patients with TB sequelae in a single lung

\begin{tabular}{|c|c|c|c|c|c|c|c|c|}
\hline \multirow[t]{2}{*}{ Parameters } & \multicolumn{4}{|c|}{ TB sequelae involved lung } & \multicolumn{4}{|c|}{ Contralateral lung } \\
\hline & $\begin{array}{l}\text { Upper } \\
\text { lobe }\end{array}$ & $\begin{array}{l}\text { Middle } \\
\text { lobe }\end{array}$ & $\begin{array}{l}\text { Lower } \\
\text { lobe }\end{array}$ & $\begin{array}{l}\text { Whole } \\
\text { lung }\end{array}$ & $\begin{array}{l}\text { Upper } \\
\text { lobe }\end{array}$ & $\begin{array}{l}\text { Middle } \\
\text { lobe }\end{array}$ & $\begin{array}{l}\text { Lower } \\
\text { lobe }\end{array}$ & $\begin{array}{l}\text { Whole } \\
\text { lung }\end{array}$ \\
\hline Bronchiectasis prevalence, $\mathrm{n}(\%)$ & $25(38.5)^{* *}$ & $21(32.3)^{*}$ & $20(30.8)$ & $39(60.0)^{* *}$ & $7(10.8)$ & $10(15.4)$ & $13(20.0)$ & $20(30.8)$ \\
\hline Smith score ${ }^{a}$ & $2.36(1.03)$ & $2.86(1.01)$ & $2.96(0.94)$ & $4.56(3.15)$ & $2.7 I(1.25)$ & $2.90(1.19)$ & $2.49(1.08)$ & $4.02(3.32)$ \\
\hline \multicolumn{9}{|l|}{ Bhalla score, ${ }^{\mathrm{b}} \mathrm{n}(\%)$} \\
\hline I & $25(100)$ & $20(95.2)$ & $18(90.0)$ & $36(92.3)$ & $7(100)$ & $7(70.0)$ & $12(92.3)$ & $17(85.0)$ \\
\hline$\geq 2$ & $0(0)$ & $\mathrm{I}(4.8)$ & $2(10.0)$ & $3(7.7)$ & $0(0)$ & $3(30.0)$ & I (7.7) & $3(15.0)$ \\
\hline Emphysema prevalence, n (\%) & $35(53.8)$ & $23(35.4)$ & $30(46.2)$ & $39(60.0)$ & $32(49.2)$ & $21(32.3)$ & $28(43.1)$ & $35(53.8)$ \\
\hline Centrilobular & $21(32.3)$ & $7(10.8)$ & $13(20.0)$ & $25(38.5)$ & $18(27.7)$ & $10(15.4)$ & $13(20.0)$ & $21(32.3)$ \\
\hline Panlobular & $17(26.2)$ & $9(13.8)$ & $14(21.5)$ & $25(38.5)$ & $13(20.0)$ & $5(7.7)$ & $13(20.0)$ & $19(29.2)$ \\
\hline Paraseptal & $3(4.6)$ & $5(7.7)$ & $5(7.7)$ & $12(18.5)$ & $4(6.2)$ & $4(6.2)$ & $3(4.6)$ & $8(12.3)$ \\
\hline Bullae & $10(15.4)$ & $6(9.2)$ & $7(10.8)$ & $17(26.2)$ & $9(13.8)$ & $3(4.6)$ & $5(7.7)$ & $13(20.0)$ \\
\hline Goddard score ${ }^{a}$ & & & & $4.28(2.70)$ & & & & $4.20(2.7 I)$ \\
\hline
\end{tabular}

Notes: *Compared with "contralateral lung", $p<0.05$; **Compared with "contralateral lung", $p<0.0$ I; aSmith score and Goddard score represent the means of patients with bronchiectasis, or with emphysema; bdefined based on the highest Bhalla score of the patient. Lingular segment of left lung was taken as the "middle lobe" of the left lung. Continuous data are expressed as mean (standard deviation); Categorical data are expressed as number (percentage).

Abbreviation: TB, tuberculosis. 


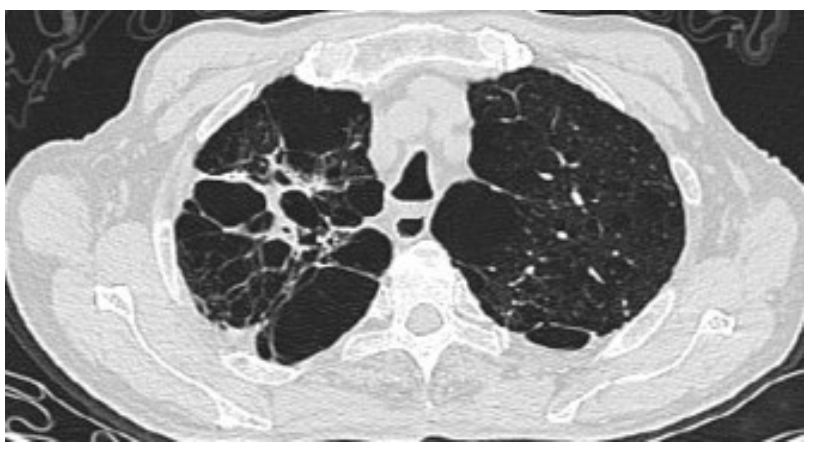

Figure 2 Similar emphysema at LUL and RUL in case 2 with TB sequelae in RUL (HRCT).

Abbreviations: LUL, left upper lobe; RUL, right upper lobe; TB, tuberculosis; HRCT, high-resolution CT.

previous PTB showed higher prevalence and more severe bronchiectasis, increased prevalence of lower lung emphysema, and more extensive emphysema, which were evident in lobes near the local sequelae of PTB and both the lungs, and this could not be explained only by the local effects of TB. Studies have shown that even localized PTB might be a risk factor for COPD, ${ }^{9,14,21}$ suggesting a role beyond its local effects. Our results provide new data to support the implication of PTB in the development and/or manifestation of COPD associated with cigarette smoking or biomass fuel exposure.

CT bronchiectasis, mostly mild-to-moderate in degree, and found in as high as $57.2 \%$ of COPD patients, was associated with exacerbations, poor lung function, and even mortality from the disease. ${ }^{17}$ The potential mechanisms underlying the development of bronchiectasis in COPD are poorly understood. Our recent study showed that previous PTB was an independent risk factor for coexistent bronchiectasis in COPD,${ }^{11}$ suggesting that PTB might be a cause of bronchiectasis in these patients. In the current study, we found that previous PTB was associated with more prevalent and severe bronchiectasis, which was more evident in the TB-involved lung, indicating local airway damage induced by TB infection.

Another interesting finding of our study was the association of previous PTB with higher prevalence of lower lung emphysema and more severe emphysema of both lungs, characterized by more extensive centrilobular emphysema, and higher prevalence of panlobular emphysema and bullae. The association of PTB with emphysema was observed as early as the 1950s. These earlier studies found increased incidence of obstructive ventilatory dysfunction by lung function testing,,$^{22,23}$ and of emphysema by chest X-ray, ${ }^{24,25}$ although the latter was not consistently confirmed by pathological studies. ${ }^{26}$ The advent of HRCT made the observation and classification of emphysema easier and more accurate, but few studies have been carried out to re-examine the association of PTB with emphysema using HRCT in cross-sectional, or ideally, longitudinal studies. Our results warrant further studies to elucidate the effect of PTB on the development of emphysema, particularly in the context of cigarette smoke or biomass fuel exposure.

The potential effect of TB on the development of COPD was believed to be mediated by mechanisms of chronic inflammation. ${ }^{27-29}$ Chronic inflammatory response may persist in patients with TB even after the microbiological cure. ${ }^{30}$ Radovic et $\mathrm{al}^{31}$ demonstrated that after 6 months of anti-TB treatment, serum markers of systemic inflammation such as erythrocyte sedimentation rate and fibrinogen decreased significantly, but were still abnormally high. ${ }^{31}$ As early as 1960 s, Hennes et al ${ }^{32}$ showed that antibodies reacting with extracts of human lungs were present in the serum of many patients with PTB and idiopathic obstructive emphysema, suggesting that tubercle bacilli might serve as an "endogenous Freund adjuvant" stimulating antibody response to damaged tissue. ${ }^{32}$ Recently, Tang et $\mathrm{l}^{33}$ found that serum
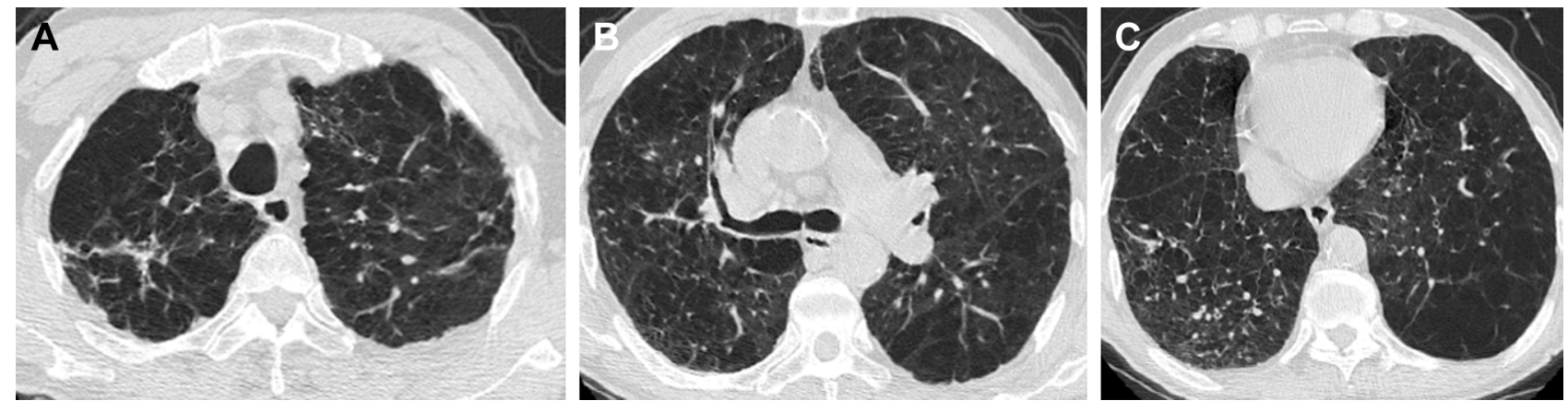

Figure 3 Extensive emphysema and mild bronchiectasis in case 3 with TB sequelae at RUL (HRCT).

Notes: (A) upper level; (B) middle level; (C) lower level.

Abbreviations: TB, tuberculosis; RUL, right upper lobe; HRCT, high-resolution CT. 
concentrations of cytokines such as soluble interleukin-2 receptor (sIL-2R), IL-6 and tumor necrosis factor (TNF- $\alpha$ ) in COPD patients with TB were higher than those without TB or those with TB but without COPD, ${ }^{33}$ suggesting that COPD combined with TB may result in excessive inflammation. Studies on airway or lung inflammation, such as with bronchoalveolar lavage (BAL), in patients with PTB showed that expressions of matrix metalloproteinases (MMPs) such as matrix metalloproteinase-1, -3 and -9 (MMP-1, MMP-3 and MMP-9), which played important roles in tissue destruction, were significantly upregulated when compared with normal or symptomatic controls. ${ }^{34,35}$ BAL concentrations of proinflammatory cytokines such as TNF- $\alpha$, IL-6 and IL-8, were also found to be correlated with the HRCT score in active pulmonary TB. ${ }^{36}$ However, the features of airway/lung inflammation in COPD patients with previous PTB need to be clarified further.

Previous PTB in COPD patients was also clinically relevant as these patients complained of more significant dyspnea and had more severe exacerbations. In our study, no significant difference was found in spirometry measurements ( $\mathrm{FEV}_{1} / \mathrm{FVC}$ and $\mathrm{FEV}_{1} \%$ predicted) between COPD patients with or without PTB, which was similar to the results of Yakar et al. ${ }^{37}$ Given that $\mathrm{FEV}_{1}$ correlated poorly with symptoms, impairment of a patient's quality of life, and exacerbations, ${ }^{4}$ this result could be explained, at least partially, by the presence of more prevalent and severe bronchiectasis and emphysema. ${ }^{11,18,38}$ Other results of clinical relevance include the finding that positive culture of $P$. aeruginosa in sputum was seen mostly in COPD patients with prior PTB, which could be explained by the presence of more severe bronchiectasis in this group of patients. Therefore, in COPD patients with prior PTB, especially those with coexistent bronchiectasis, regular sputum surveillance for $P$. aeruginosa, and strategies for eradication of the pathogen may be useful for prevention of COPD exacerbations. Because previous PTB was also associated with unique features of emphysema, interventional treatments such as volume reduction may need special consideration; for example, in volume reduction surgery, COPD patients with prior PTB may experience less post-treatment pneumothorax due to pleural adhesions.

It should be noted that, in our study, typical TB sequelae on chest CT was the only criterion for the diagnosis of prior PTB in the majority of our patients. This diagnostic criterion has been used in several studies, ${ }^{11-13}$ especially in high TB-burden countries. . $^{97,39}$ However, prior PTB cannot be completely excluded if no TB sequelae are found by chest X-ray or CT. Our study also has several limitations.
Because of the cross-sectional design of the study, the time sequence of the occurrence of PTB and emphysema and/or bronchiectasis cannot be determined, and therefore, a causeeffect relationship cannot be obtained. No evaluation of the interaction between PTB and cigarette smoke was attempted, though the smoking status of the TB group and the non-TB group of our patients was similar. We believe that this interaction would be very important for understanding the potential effect of PTB in COPD.

\section{Conclusion}

We have found in a well-defined cohort of COPD patients that those with previous PTB had more prevalent and severe bronchiectasis and emphysema on HRCT, which were associated with significant dyspnea and higher frequency of severe exacerbations. Although PTB may have a local effect on bronchiectasis, its involvement in airspace damage of COPD may be extensive. Further prospective studies of larger sample size are warranted to clarify the effects and mechanisms of PTB on the development or progression of COPD, particularly in the context of cigarette smoking.

\section{Acknowledgment}

The authors thank Lijin Lu, Ying Zhang, Yang Wang, Xiufang Luo, Jie Zhuo, Dongning Chen, Yong Liu, Peng Bai, Ran Li, Yuhong Wang and Haiyan Sheng for their support in screening subjects participating in the study. The authors acknowledge financial support from National Natural Science Foundation of China (81170039, 81470239), Highlevel Talent Training Foundation of Beijing Health System (2014-3-011), and Beijing Talent Training Foundation (No 2009D003003000002).

\section{Ethics approval and consent to participate}

Written informed consent was obtained from all the patients, and the study was approved by the local Ethics Committee of Beijing Tongren Hospital, Capital Medical University.

\section{Authors' contribution}

All authors contributed toward data analysis, drafting and critically revising the paper, and approved the final version of the manuscript. JJ completed the recruitment of patients, performed the collection and analysis of all data, and was a major contributor to the study design and manuscript. YS was the primary investigator of this study and a major contributor to the study design and manuscript writing. WY and SL were mainly responsible for image evaluation. XL mainly 
completed the recruitment of patients, and performed the collection of clinical data.

\section{Disclosure}

The authors report no conflicts of interest in this work.

\section{References}

1. Sun YC. A dangerous combination: tuberculosis and chronic obstructive pulmonary disease. Chin Med J (Engl). 2013;126(12):2203-2204.

2. Inghammar M, Ekbom A, Engström G, et al. COPD and the risk of tuberculosis-a population-based cohort study. PLoS One. 2010;5(4): e10138.

3. van Zyl Smit RN, Pai M, Yew WW, et al. Global lung health: the colliding epidemics of tuberculosis, tobacco smoking, HIV and COPD. Eur Respir J. 2010;35(1):27-33.

4. Global initiative for chronic obstructive lung disease, global strategy for the diagnosis, management, and prevention of chronic obstructive pulmonary disease; 2017. Available from: http://www.goldcopd. org/2017. Accessed November 2016.

5. Menezes AM, Hallal PC, Perez-Padilla R, et al; Latin American Project for the Investigation of Obstructive Lung Disease (PLATINO) Team. Tuberculosis and airflow obstruction: evidence from the PLATINO study in Latin America. Eur Respir J. 2007;30(6):1180-1185.

6. Hnizdo E, Singh T, Churchyard G. Chronic pulmonary function impairment caused by initial and recurrent pulmonary tuberculosis following treatment. Thorax. 2000;55(1):32-38.

7. Caballero A, Torres-Duque CA, Jaramillo C, et al. Prevalence of COPD in five Colombian cities situated at low, medium, and high altitude (PREPOCOL study). Chest. 2008;133(2):343-349.

8. Amaral AF, Coton S, Kato B, et al; BOLD Collaborative Research Group. Tuberculosis associates with both airflow obstruction and low lung function: BOLD results. Eur Respir J. 2015;46(4):1104-1112.

9. Jung JW, Choi JC, Shin JW, Kim JY, Choi BW, Park IW. Pulmonary Impairment in Tuberculosis Survivors: the Korean National Health and Nutrition Examination Survey 2008-2012. PLoS One. 2015; 10(10):e0141230.

10. Martinez-Garcia MA, Miravitlles M. Bronchiectasis in COPD patients: more than a comorbidity? Int J COPD. 2017;12:1401-1411.

11. Jin J, Yu W, Li S, Lu L, Liu X, Sun Y. Factors associated with bronchiectasis in patients with moderate-severe chronic obstructive pulmonary disease. Medicine (Baltimore). 2016;95(29):e4219.

12. Lam KB, Jiang CQ, Jordan RE, et al. Prior TB, smoking, and airflow obstruction: a cross-sectional analysis of the Guangzhou Biobank Cohort Study. Chest. 2010;137(3):593-600.

13. Kim HJ, Baek S, Kim HJ, et al. The impact of smoking on airflow limitation in subjects with history of asthma and inactive tuberculosis. PLoS One. 2015;10:e0125020.

14. Hansell DM, Bankier AA, MacMahon H, McLoud TC, Müller NL, Remy J. Fleischner society: glossary of terms for thoracic imaging. Radiology. 2008;246(3):697-722.

15. Smith IE, Jurriaans E, Diederich S, Ali N, Shneerson JM, Flower CD. Chronic sputum production: correlations between clinical features and findings on high resolution computed tomographic scanning of the chest. Thorax. 1996;51(9):914-918.

16. Gallego M, Pomares X, Espasa M, et al. Pseudomonas aeruginosa isolates in severe chronic obstructive pulmonary disease: characterization and risk factors. BMC Pulmon Med. 2014;14:103.

17. Martínez-García MA, de la Rosa Carrillo D, Soler-Cataluña JJ, et al. Prognostic value of bronchiectasis in patients with moderate-to-severe chronic obstructive pulmonary disease. Am J Respir Crit Care Med. 2013;187(8):823-831.
18. Bhalla M, Turcios N, Aponte V, et al. Cystic fibrosis: scoring system with thin-section CT. Radiology. 1991;179(3):783-788.

19. Goddard PR, Nicholson EM, Laszlo G, Watt I. Computed tomography in pulmonary emphysema. Clin Radiol. 1982;33(4):379-387.

20. Makita H, Nasuhara Y, Nagai K, et al; Hokkaido COPD Cohort Study Group. Characterisation of phenotypes based on severity of emphysema in chronic obstructive pulmonary disease. Thorax. 2007; 62(11):932-937.

21. Harries AD, Ade S, Burney P, Hoa NB, Schluger NW, Castro JL. Successfully treated but not fit for purpose: paying attention to chronic lung impairment after TB treatment. Int J Tuberc Lung Dis. 2016; 20(8):1010-1014.

22. Lancaster JF, Tomashefski JF. Tuberculosis-a cause of emphysema. Am Rev Respir Dis. 1963;87:435-437.

23. Anno H, Tomashefski JF. Studies on the impairment of respiratory function in pulmonary tuberculosis. Am Rev Tuberc. 1955;71(3, Part 1): 333-348.

24. Singh D, Richards WF. Obstructive emphysema in primary pulmonary tuberculosis. Tubercle. 1957;38(6):397-402.

25. Matsaniotis N, Kattamis C, economou-Mavrou E, Kyriazakou M. Bullous emphysema in childhood tuberculosis. J Pediatr. 1967;71(5): 703-708.

26. Martin CJ, Cochran TH, Katsura S. Tuberculosis, emphysema, and bronchitis. Am Rev Respir Dis. 1968;97(6):1089-1093.

27. de la Mora IL, Martínez-Oceguera D, Laniado-Laborín R. Chronic airway obstruction after successful treatment of tuberculosis and its impact on quality of life. Int J Tuberc Lung Dis. 2015;19(7):808-810.

28. Hwang YI, Kim JH, Lee CY, et al. The association between airflow obstruction and radiologic change by tuberculosis. J Thorac Dis. 2014; $6(5): 471-476$.

29. Jordan TS, Spencer EM, Davies P. Tuberculosis, bronchiectasis and chronic airflow obstruction. Respirology. 2010;15(4):623-628.

30. Pasipanodya JG, Miller TL, Vecino M, et al. Pulmonary impairment after tuberculosis. Chest. 2007;131(6):1817-1824.

31. Radovic M, Ristic L, Ciric Z, et al. Changes in respiratory function impairment following the treatment of severe pulmonary tuberculosis limitations for the underlying COPD detection. Int J Chron Obstruct Pulmon Dis. 2016;11:1307-1316.

32. Hennes AR, Moore MZ, Carpenter RL, Hammarsten JF. Antibodies to human lung in patients with obstructive emphysema and pulmonary tuberculosis. Am Rev Respir Dis. 1961;83:354-358.

33. Tang S, Cui H, Yao L, et al. Increased cytokines response in patients with tuberculosis complicated with chronic obstructive pulmonary disease. PLoS One. 2013;8(4):e62385.

34. Chang JC, Wysocki A, Tchou-Wong KM, Moskowitz N, Zhang Y, Rom WN. Effect of Mycobacterium tuberculosis and its components on macrophages and the release of matrix metalloproteinases. Thorax. 1996;51(3):306-311.

35. Elkington P, Shiomi T, Breen R, et al. MMP-1 drives immunopathology in human tuberculosis and transgenic mice. J Clin Invest. 2011; 121(5):1827-1833.

36. Casarini M, Ameglio F, Alemanno L, et al. Cytokine levels correlate with a radiologic score in active pulmonary tuberculosis. Am J Respir Crit Care Med. 1999;159(1):143-148.

37. Yakar HI, Gunen H, Pehlivan E, Aydogan S. The role of tuberculosis in COPD. Int J Chron Obstruct Pulmon Dis. 2017;12:323-329.

38. Alcaide AB, Sanchez-Salcedo P, Bastarrika G, et al. Clinical features of smokers with radiological emphysema but without airway limitation. Chest. 2017;151(2):358-365.

39. Allwood BW, Goldin J, Said-Hartley Q, et al. Assessment of previous tuberculosis status using questionnaires, chest X-rays and computed tomography scans. Int J Tuberc Lung Dis. 2015;19(12):1435-1440. 


\section{Publish your work in this journal}

The International Journal of COPD is an international, peer-reviewed journal of therapeutics and pharmacology focusing on concise rapid reporting of clinical studies and reviews in COPD. Special focus is given to the pathophysiological processes underlying the disease, intervention programs, patient focused education, and self management protocols.

This journal is indexed on PubMed Central, MedLine and CAS. The manuscript management system is completely online and includes a very quick and fair peer-review system, which is all easy to use. Visit http://www.dovepress.com/testimonials.php to read real quotes from published authors.

Submit your manuscript here: http://www.dovepress.com/international-journal-of-chronic-obstructive-pulmonary-disease-journal 